\title{
Effect of Bojungikki-tang on Chronic Pelvic Pain Syndrome: Case Series
}

\section{Jiman Kim ${ }^{1}$ and Seung Won Kwon ${ }^{2 *}$}

${ }^{1}$ Kyunghee-saeng Haniwon (Oriental Medicine Clinic), Seoul, South Korea

${ }^{2}$ Department of Neurologic Diseases, Kyung Hee University Oriental Medicine Hospital, Seoul, South Korea

\begin{abstract}
Background: Chronic Pelvic Pain Syndrome (CPPS) is a pelvic pain condition in men that involves urinary symptoms and sexual activity-related discomfort and should be distinguished from other forms of prostatitis such as chronic bacterial prostatitis and acute bacterial prostatitis. CPPS significantly affects men's quality of life. We experienced six cases which revealed improved CPPS symptoms after herbal medicine therapy.
\end{abstract}

Case report: We describe 6 cases of CPPS that could not bead equately controlled with antibiotics or alpha blockers. We decided to administer Bojungikki-tang, an herbal extract used in Korean medicine.

At baseline, $\mathrm{NIH}$-Chronic Prostatitis Symptom Index score of 6 cases was $28.83 \pm 6.37$ (average \pm standard deviation). After 5 months $\sim 10$ months of treatment with Bojungikki-tang, we observed a reduction of CPPS symptoms such as pelvic pain, frequent urination, and sexual symptoms. Among 6 cases, 5 cases reveals $\mathrm{NIH}$ Chronic Prostatitis Symptom Index score 0 point (83.3\%), 1 case reveals 14 points after herbal medication treatment.

Discussion: Our case series has shown that the symptoms of CPPS may be controlled using herbal medication when unresponsive to conventional therapies.

Keywords: Bojungikki-tang; Chronic pelvic pain syndrome; Herbal medicine; Chronic nonbacterial prostatitis; CPPS

\section{Introduction}

Chronic pelvic pain syndrome (CPPS) is a pelvic pain condition in men that involves urinary symptoms and sexual activity-related discomfort, and should be distinguished from other forms of prostatitis such as chronic bacterial prostatitis and acute bacterial prostatitis [1]. CPPS presents as pelvic or perineal pain, frequent urination, increased urgency, abdominal pain, constant burning pain in the penis, and postejaculatory pain without urinary tract infection lasting longer than 3 months.

However, CPPS is a poorly understood disorder and no definitive diagnostic tests exist. The cause of CPPS is also uncertain. Therefore, this disorder is difficult to treat [2]. Oral antibiotics and alpha blockers are currently used as pharmacological therapy in CPPS [35]. Psychological treatment approaches and physical therapies such as massage have also been used. However, the efficacy of these treatment approaches is relatively low. Therefore, additional therapies that can alleviate the symptoms of CPPS and improve quality of life (QOL) are necessary.

We observed a positive effect of Bojungikki-tang (BJGT), an herbal extract used in Korean medicine, on CPPS symptoms in patients who were unresponsive to conventional therapies such as antibiotics and alpha blockers.

\section{Case History}

\section{Methods and intervention}

We selected patients with pelvic pain, frequent urination, and sexual activity-related symptoms due to CPPS. The diagnosis of CPPS was based on previous urine analysis, semen, and prostatic secretions tests which reveals normal values and did not indicate infection as well as patient-reported symptoms. We selected patients who also showed symptoms of "Qi deficiency," a concept in traditional Korean medicine. Qi deficiency is a syndrome that consists of symptoms such as chronic fatigue, dyspepsia, anorexia, general weakness, immune system dysfunction, depressive mood, and/or chronic sleep disorder. We attempted to treat 6 CPPS patients with the herbal complex BJGT, which has been used to treat Qi deficiency in traditional Korean Medicine.

Patients were treated with BJGT (3 times/day) only for a period of approximately 6 months. The daily dose of BJGT consisted of Astragalus membranaceus (6 g), Panax ginseng, Glycyrrhizae radix, Atractylodes japonica (4 g), Citrus unshiu, Angelica gigas (3 g), Cimicifuga heracleifolia, and Bupleurum falcatum (2 g).

\section{History and treatment outcomes}

Case 1: A 36-year-old man $(176 \mathrm{~cm}, 61 \mathrm{~kg})$ with CPPS presented at the Kyunghee-saeng Korean Medicine Clinic. He was diagnosed with CPPS in January 2012. He experienced perineal and pubic pain with a visual analogue scale (VAS) pain score of 6 , frequent urination, and a sensation of incomplete bladder emptying. After diagnosis, he was treated with antibiotics for 3 months, but his symptoms remained unchanged as of June 2012. The patient elected to treat his pelvic and urinary symptoms with Korean herbal medicine. His body type was thin, he had a tendency to feel dizziness easily, and he had chronic insomnia, chronic dyspepsia, and fatigue, which are all symptoms of Qi deficiency syndrome. BJGT treatment was therefore initiated. At the start of herbal medicine therapy, the patient's NIH-Chronic Prostatitis Symptom Index (NIH-CPSI) score was 32. Three months later, his

*Corresponding author: Seung Won Kwon, Department of Cardiovascular and Neurologic Diseases, Kyung Hee University Oriental Medicine Hospital, 1 Hoegidong, Dongdaemun-gu, Seoul 130-702, South Korea, Tel: 82-02-958-9128; Fax: 82-02-958-9132; E-mail: kkokkottung@hanmail.net

Received November 06, 2013; Accepted November 22, 2013; Published November 25, 2013

Citation: Kim J, Kwon SW (2013) Effect of Bojungikki-tang on Chronic Pelvic Pain Syndrome: Case Series. Altern Integ Med 2: 146. doi:10.4172/2327-5162.1000146

Copyright: (c) $2013 \mathrm{Kim} \mathrm{J}$, et al. This is an open-access article distributed under the terms of the Creative Commons Attribution License, which permits unrestricted use, distribution, and reproduction in any medium, provided the original author and source are credited. 
NIH-CPSI score had decreased to 28, and 6 months after the start of treatment, his NIH-CPSI score was 0. After 6 months of BJGT therapy, the patient's previous symptoms were completely eliminated, and his accompanied symptoms which were belongs to "Qi deficiency" were also reduced. The results of the follow-up measurements are provided in Figure 1.

Case 2: A 30-year-old man $(172 \mathrm{~cm}, 63 \mathrm{~kg})$ was diagnosed with CPPS in December 2010. He experienced perineal and pubic pain with a VAS pain score of 6, burning sensation during urination, discomfort after sexual climax, painful ejaculation, frequent urination (2 times per hour), and a sensation of incomplete bladder emptying. After diagnosis, he was treated with NSAIDs and antibiotics for 4 months. However, his symptoms remained unchanged as of December 2012. He also had a thin body type and experienced chronic insomnia, fatigue, chronic dyspepsia, and depressive and nervous mood changes due to the CPPS symptoms. Based on his combined symptoms, he was diagnosed with Qi deficiency, and BJGT therapy was initiated. At the start of the herbal medicine therapy, the patient's NIH-CPSI score was 36 . After 4 months, his NIH-CPSI score had decreased to 14, and 6 months after the start of treatment, his NIH-CPSI score was 0 . After 6 months of BJGT therapy, the patient's previous symptoms were completely eliminated, and his accompanied symptoms which were belongs to "Qi deficiency" were also reduced. The results of the follow-up measurements are provided in Figure 1.

Case 3: A 27-year-old CPPS patient $(170 \mathrm{~cm}, 60 \mathrm{~kg})$ was diagnosed in September 2009. He experienced pain in the pubic region and tip of the penis with a VAS pain score of 6 , burning sensation during urination, discomfort after sexual climax, erectile dysfunction, loss of sexual desire, frequent urination, and a sensation of incomplete bladder emptying. After diagnosis, he was treated with NSAIDs and antibiotics for 3 months. Over the course of 2 years, the patient explored various treatments including acupuncture, moxibustion, corrective pelvic osteotomy, and bee venom therapy. However, his symptoms remained unchanged as of September 2012. He also had a thin body type and experienced chronic fatigue, dyspepsia, difficulty concentrating, and depressive mood changes due to CPPS symptoms, indicating Qi deficiency. BJGT therapy was therefore initiated. At the start of herbal medicine therapy, his NIH-CPSI score was 25. After 6 months, his NIH-CPSI score was 0 . After 6 months of BJGT therapy, the patient's previous symptoms were completely eliminated, and his accompanied symptoms which were belongs to "Qi deficiency" were also reduced. The results of the follow-up measurements are provided in Figure 1.

Case 4: A 46-year-old man $(178 \mathrm{~cm}, 63 \mathrm{~kg})$ who was diagnosed with CPPS in 2002 presented to the Kyunghee-saeng Korean Medicine Clinic to receive herbal medicine therapy for his CPPS symptoms. His symptoms were perineal and pubic pains with a VAS pain score of 7 that was aggravated in the seated position, frequent urination, and a sensation of incomplete bladder emptying. After diagnosis, he was treated with antibiotics and an alpha blocker for 3 months. When he first presented at our clinic, he was only taking an alpha blocker. However, the patient's symptoms had not cleared as of October 2012. He also had a thin body type and experienced chronic fatigue and dyspepsia, indicating Qi deficiency. BJGT treatment was therefore initiated. At the start of herbal medicine therapy, his NIH-CPSI score was 34 . After 6 months, his NIH-CPSI score had decreased to 13. After 6 months of BJGT therapy, the patient's pelvic pain was reduced (VAS 2) and his urinary symptoms were also decreased. His accompanied symptoms which were belongs to "Qi deficiency" were also reduced. The results of the follow-up measurements are provided in Figure 1.

Case 5: A 26-year-old man $(168 \mathrm{~cm}, 59 \mathrm{~kg})$ presented at our clinic to receive herbal medicine therapy for his CPPS symptoms. He was diagnosed with CPPS in September 2012. The patient experienced perineal and testicular pains with a VAS pain score of 4 . He was not able to wear skinny jeans because of his testicular pain. After diagnosis, he was treated with antibiotics for 3 months. However, his symptoms had not cleared as of December 2012. When he first presented at our clinic, he was no longer taking antibiotics. He also had a thin body type and experienced chronic fatigue and dyspepsia, indicating Qi deficiency. BJGT therapy was therefore initiated. At the start of herbal medicine therapy, his NIH-CPSI score was 19. After 3 months, the patient's NIH-CPSI score had decreased to 14. Six months after starting therapy, his NIH-CPSI score was 7, and 10 months after starting therapy, all his symptoms were eliminated. He accompanied symptoms which were

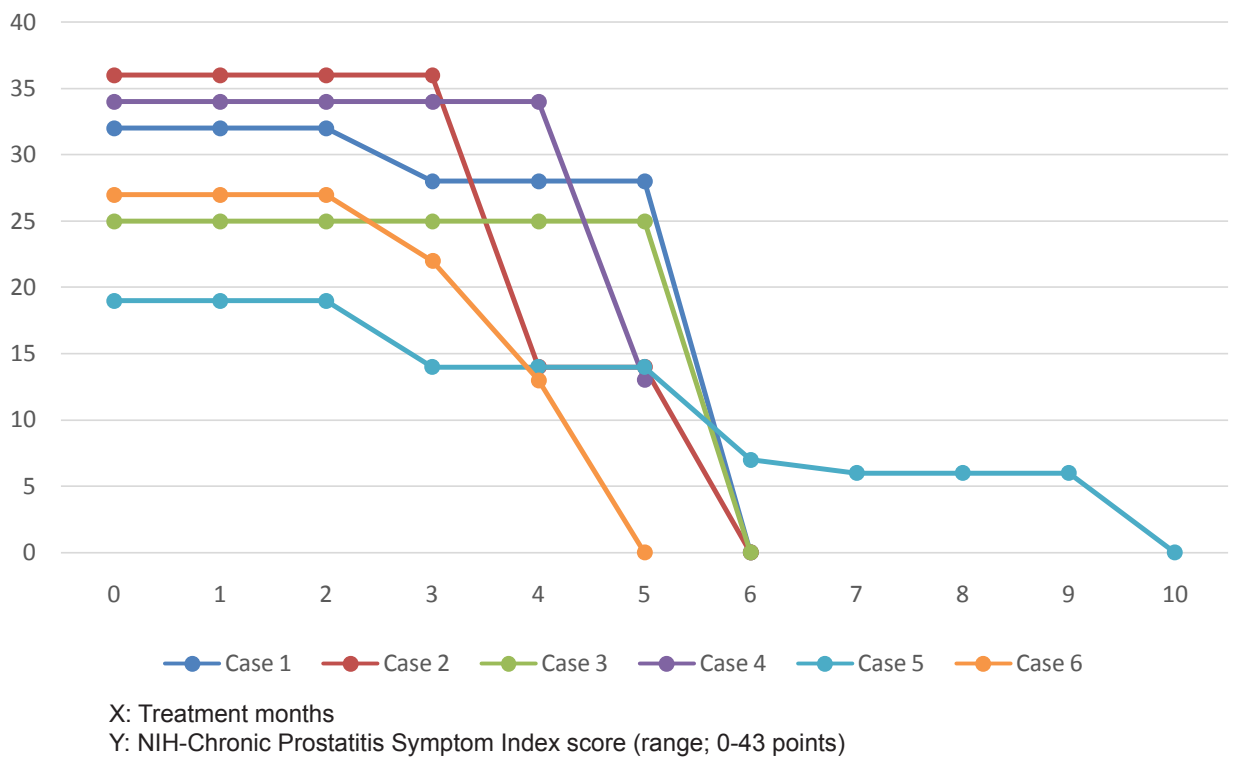

Figure 1: Change in the $\mathrm{NIH}-$ Chronic Prostatitis Symptom Index score in case 1 6. 
belongs to "Qi deficiency" were also reduced. The results of follow-up measurements are provided in Figure 1.

Case 6: A 26-year-old man $(169 \mathrm{~cm}, 58 \mathrm{~kg})$ was diagnosed with CPPS in 2005. He experienced pain in the perineum, testicle, and tip of the penis with a VAS pain score of 4 , burning sensation during urination, frequent urination, and a sensation of incomplete bladder emptying. After diagnosis, he was treated with antibiotics for 1 year. However, there was no clear response as of May 2013. The patient then presented at our clinic to receive herbal medicine therapy for his CPPS symptoms. He also had a thin body type and experienced chronic fatigue, dyspepsia, and cold hands and feet. On the basis of these symptoms, we decided that he was in the Qi deficiency state, and BJGT therapy was therefore initiated. At the start of the herbal medicine therapy, his NIH-CPSI score was 27. After 3 months, his NIH-CPSI score had decreased to 22, 4 months after the start of the treatment, his NIH-CPSI score was 13, and 6 months after the start of the treatment, all his symptoms were eliminated. The patient's accompanied symptoms were also reduced. The results of the followup measurements are provided in Figure 1.

\section{Discussion}

In the present 6-case series, we showed that a reduction of CPPSassociated pelvic pain, urinary symptoms, and sexual activity-related symptoms that were unresponsive to oral antibiotics, NSAIDs, and alpha blockers could be achieved by approximately 6 months of treatment with BJGT.

Our case series has important implications. First, symptoms of CPPS that were unresponsive to conventional therapies were reduced by the herbal medicine BJGT. CPPS is difficult to treat. In conventional therapy, alpha blockers, antibiotics, and NSAIDs have been used to treat CPPS symptoms. However, antibiotics therapy is controversial. Several studies suggest that antibiotics are beneficial in reducing symptoms [3], while others have questioned the utility of antibiotics in CPPS [4]. A meta-analysis showed that alpha blockers (tamsulosin and alfuzosin) were beneficial when the duration of therapy was at least 3 months [5]. In addition, there are other therapeutic approaches to CPPS, such as physical therapy (massage), psychological therapy, acupuncture, and transurethral needle ablation of the prostate that proved ineffective in clinical trials [6]. In the present case series, all the patients had used antibiotics, NSAIDs, or alpha blockers for at least 3 months. In addition, case 3 had also used acupuncture and moxibustion therapy. However, in all the cases, CPPS symptoms did not respond to conventional therapies. After 6 months of BJGT treatment, the symptoms were eliminated in 5 patients and reduced in the remaining patient.

We were also able to improve the CPPS patients' quality of life with BJGT. We used the NIH-CPSI score, which includes a category that assesses patient QOL, to evaluate the effects of BJGT on CPPS. After the BJGT treatment, NIH-CPSI QOL scores were 0 in 5 cases and decreased from 9 to 2 in the remaining case. Accompanying symptoms such as chronic insomnia, chronic fatigue and depressive mood changes, which can impact patients' QOL, were also eliminated in all the cases. Recently, BJGT has been used for improving cancer patients' QOL and fatigue. Jeong et al. [7] suggested that BJGT may have beneficial effects on fatigue and QOL in cancer patients. Another study also suggested that BJGT can improve QOL-related symptoms such as fatigue, depressive mood, and anorexia, which occur in cancer patients on chemotherapy [8]. We believe that the BJGT-associated improvement of QOL observed in these CPPS cases is in this context.
We assume that the mechanism of the anti-chronic prostatitis effect of BJGT might be immune modulation. An interaction between psychological factors and dysfunction in the immune, neurological, and endocrine systems is assumed to be a mechanism of CPPS [9]. There are several theories behind CPPS, such as stress-induced hypothalamicpituitary-adrenalaxis or endocrine dysfunction, neurogenic inflammation, and myofascial pain syndrome [10-16]. Among these, neurogenic inflammation is assumed to be the result of pelvic nervous system dysregulation due to past infection, pelvic trauma, anxiety, or chronic unconscious pelvic tension. This dysregulation can lead to inflammation mediated by substances such as substance $\mathrm{P}$ released by nerve cells. In addition, the prostate can become inflamed owing to the action of the chronically activated pelvic nerves on the mast cells at the end of the nerve. An experimental study confirmed stress-induced genitourinary inflammation in rat models [17]. BJGT has been widely known to improve immunosuppressive states $[18,19]$, reinforce natural killer (NK) cell activity [20], and protect against viral infection [21-27]. We believe that these immunomodulatory effects of BJGT have played a role in the present case series.

"Qi deficiency" consists of several symptoms such as fatigue, anorexia, diarrhea, dyspepsia, sleep disturbance, myalgia, impaired memory or concentration, depressed immunofucntion, depressive mood, nocturnal urination, urinary incontinence, proctoptosis, and hemorrhoid. These symptoms of "Qi deficiency" in Korean medicine are similar to the chronic fatigue syndrome. Several studies have suggested that the mechanism of the chronic fatigue syndrome can be related to oxidative stress, genetic condition, infection, hypothalamicpituitary-adrenal axis abnormalities, immune dysfunction and psychological factors [28]. BJGT revealed immunomodulatory effect on chronic fatigue syndrome models in some studies [29-31]. Therefore, we could assume that the effect of BJGT on "Qi deficiency" is also an immunomodulation. All patients in present cases revealed frequent urination, pelvic pain and other symptoms which are revealed in "Qi deficiency". Furthermore, there are several theories behind CPPS, such as stress-induced hypothalamic-pituitary-adrenal axis dysfunction, neurogenic inflammation which are similar to theories behind chronic fatigue syndrome. Therefore, we selected BJGT which has an immonomudulatory effect to control symptoms of CPPS and "Qi deficiency".

Among the constitutions of BJGT, Panax ginseng has a prevent effect on chronic bacterial prostatitis [32]. Although effect of Panax ginseng is refer to chronic bacterial prostatitis prevention, we think that Panax ginseng make a main role in immunomudulatory function of BJGT.

We showed positive clinical effects of BJGT on symptom relief in 6 patients with unresponsive CPPS. However, this is only a case series and our herbal medication does not have Good Manufacturing Practice certification. Furthermore, present study is just case series, not randomized controlled design. Therefore, to confirm the clinical effects of the extract, a clinical study involving a control group and a standardized medication is needed.

\section{References}

1. Schaeffer AJ, Datta NS, Fowler JE Jr, Krieger JN, Litwin MS, et al. (2002) Overview summary statement. Diagnosis and management of chronic prostatitis/chronic pelvic pain syndrome (CP/CPPS). Urology 60: 1-4.

2. Potts J, Payne RE (2007) Prostatitis: Infection, neuromuscular disorder, or pain syndrome? Proper patient classification is key. Cleve Clin J Med 74 Suppl 3: S63-S71.

3. Anothaisintawee T, Attia J, Nickel JC, Thammakraisorn S, Numthavaj P, et 
al. (2011) Management of chronic prostatitis/chronic pelvic pain syndrome: a systematic review and network meta-analysis. JAMA 305: 78-86.

4. Wagenlehner FM, Naber KG, Bschleipfer T, Brähler E, Weidner W (2009) Prostatitis and male pelvic pain syndrome: diagnosis and treatment. Dtsch Arztebl Int 106: 175-183.

5. Yang G, Wei Q, Li H, Yang Y, Zhang S, et al. (2006) The effect of alphaadrenergic antagonists in chronic prostatitis/chronic pelvic pain syndrome: a meta-analysis of randomized controlled trials. J Androl 27: 847-852.

6. Leskinen MJ, Kilponen A, Lukkarinen O, Tammela TL (2002) Transurethral needle ablation for the treatment of chronic pelvic pain syndrome (category III prostatitis): a randomized, sham-controlled study. Urology 60: 300-304.

7. Jeong JS, Ryu BH, Kim JS, Park JW, Choi WC, et al. (2010) Bojungikki-tang for cancer-related fatigue: a pilot randomized clinical trial. Integr Cancer Ther 9: 331-338.

8. Ishiura $Y$, Yamamoto H, Shiba $Y$, Terasaki $Y$, Ishida $Y$, et al. (2013) [Effect of Japanese traditional medicine, TJ-41, on quality of life of patients with nonsmall cell lung cancer receiving outpatient chemotherapy]. Gan To Kagaku Ryoho 40: 913-916.

9. Pontari MA, Ruggieri MR (2008) Mechanisms in prostatitis/chronic pelvic pain syndrome. J Urol 179: S61-S67.

10. Anderson RU, Orenberg EK, Chan CA, Morey A, Flores V (2008) Psychometric profiles and hypothalamic-pituitary-adrenal axis function in men with chronic prostatitis/chronic pelvic pain syndrome. J Urol 179: 956-960

11. Dimitrakov J, Joffe HV, Soldin SJ, Bolus R, Buffington CA, et al. (2008) Adrenocortical hormone abnormalities in men with chronic prostatitis/chronic pelvic pain syndrome. Urology 71: 261-266.

12. Theoharides TC, Cochrane DE (2004) Critical role of mast cells in inflammatory diseases and the effect of acute stress. J Neuroimmunol 146: 1-12.

13. Theoharides TC, Kalogeromitros D (2006) The critical role of mast cells in allergy and inflammation. Ann N Y Acad Sci 1088: 78-99.

14. Sant GR, Kempuraj D, Marchand JE, Theoharides TC (2007) The mast cell in interstitial cystitis: role in pathophysiology and pathogenesis. Urology 69: 34-40.

15. Anderson RU, Wise D, Sawyer T, Chan C (2005) Integration of myofascial trigger point release and paradoxical relaxation training treatment of chronic pelvic pain in men. J Urol 174: 155-160

16. Anderson RU, Wise D, Sawyer T, Chan CA (2006) Sexual dysfunction in men with chronic prostatitis/chronic pelvic pain syndrome: improvement after trigger point release and paradoxical relaxation training. J Urol 176: 1534-1538.

17. Alexacos N, Pang X, Boucher W, Cochrane DE, Sant GR, et al. (1999) Neurotensin mediates rat bladder mast cell degranulation triggered by acute psychological stress. Urology 53: 1035-1040

18. Utsuyama M, Seidlar H, Kitagawa M, Hirokawa K (2001) Immunological restoration and anti-tumor effect by Japanese herbal medicine in aged mice. Mech Ageing Dev 122: 341-352.

19. Kimura M, Sasada T, Kanai M, Kawai Y, Yoshida Y, et al. (2008) Preventive effect of a traditional herbal medicine, Hochu-ekki-to, on immunosuppression induced by surgical stress. Surg Today 38: 316-322.

20. Cho S, Hong T, Kaneko A, Yoshino G, Sato N, et al. (2004) Evaluation of immunological effects of hochu-ekki-to (TJ-41) prophylactic administration in mice. Am J Chin Med 32: 235-243.

21. Mori K, Kido T, Daikuhara H, Sakakibara I, Sakata T, et al. (1999) Effect of Hochu-ekki-to (TJ-41), a Japanese herbal medicine, on the survival of mice infected with influenza virus. Antiviral Res 44: 103-111.

22. Hossain MS, Takimoto H, Hamano S, Yoshida H, Ninomiya T, et al. (1999) Protective effects of hochu-ekki-to, a Chinese traditional herbal medicine against murine cytomegalovirus infection. Immunopharmacology 41: 169-181.
23. Kido T, Mori K, Daikuhara H, Tsuchiya H, Ishige A, et al. (2000) The protective effect of hochu-ekki-to (TJ-41), a Japanese herbal medicine, against HSV-1 infection in mitomycin C-treated mice. Anticancer Res 20: 4109-4113.

24. Yamaoka Y, Kawakita T, Nomoto $K$ (2000) Protective effect of a traditiona Japanese medicine, Bu-zhong-yi-qi-tang (Japanese name: Hochu-ekki-to), on the restraint stress-induced susceptibility against Listeria monocytogenes. Immunopharmacology 48: 35-42.

25. Kiyohara H, Nagai T, Munakata K, Nonaka K, Hanawa T, et al. (2006) Stimulating effect of Japanese herbal (kampo) medicine, hochuekkito on upper respiratory mucosal immune system. Evid Based Complement Alternat Med 3: 459-467.

26. Yamaya M, Sasaki T, Yasuda H, Inoue D, Suzuki T, et al. (2007) Hochu-ekki-to inhibits rhinovirus infection in human tracheal epithelial cells. $\mathrm{Br} J$ Pharmacol 150: 702-710

27. Ishih A, Nagata T, Miyase T, Ohori K, Terada M (2007) Protective effects of a kampo medicine, Hochu-ekki-to (TJ-41) on lethal malarial infection with Plasmodium chabaudi AS in A/J mice. J Nat Med 61: 280-287.

28. Patarca-Montero R, Antoni M, Fletcher MA, Klimas NG (2001) Cytokine and other immunologic markers in chronic fatigue syndrome and their relation to neuropsychological factors. Appl Neuropsychol 8: 51-64.

29. Chen R, Moriya J, Luo X, Yamakawa J, Takahashi T, et al. (2009) Hochu-ekki-to combined with interferon-gamma moderately enhances daily activity of chronic fatigue syndrome mice by increasing NK cell activity, but not neuroprotection. Immunopharmacol Immunotoxicol 31: 238-245.

30. Chen R, Moriya J, Yamakawa J, Takahashi T, Li Q, et al. (2008) Brain atrophy in a murine model of chronic fatigue syndrome and beneficial effect of Hochuekki-to (TJ-41). Neurochem Res 33: 1759-1767.

31. Wang XQ, Takahashi T, Zhu SJ, Moriya J, Saegusa S, et al. (2004) Effect of Hochu-ekki-to (TJ-41), a Japanese Herbal Medicine, on Daily Activity in a Murine Model of Chronic Fatigue Syndrome. Evid Based Complement Alternat Med 1: 203-206.

32. Kim SH, Ha US, Sohn DW, Lee SJ, Kim HW, et al. (2012) Preventive effect of ginsenoid on chronic bacterial prostatitis. J Infect Chemother 18: 709-714. 